\title{
Delegation and polarization of platforms in political competition ${ }^{\star} \equiv$
}

\author{
Ramon Faulí-Oller, ${ }^{1}$ Efe A. Ok, ${ }^{2}$ and Ignacio Ortuño-Ortín ${ }^{3}$ \\ 1 Department of Economics, University of Alicante, Campus de Sant Vicent, 03071 Alicante, SPAIN \\ (e-mail: fauli@merlin.fae.ua.es) \\ 2 Department of Economics, New York University, 269 Mercer St., New York, NY 10003, USA \\ (e-mail: efe.ok@nyu.edu) \\ 3 Department of Economics, University of Alicante and IVIE, Campus de Sant Vicent, \\ 03071 Alicante, SPAIN (e-mail: ortin@merlin.fae.ua.es)
}

Summary. We consider a model of political competition among two ideological parties who are uncertain about the distribution of voters. The distinguishing feature of the model is that parties can delegate electoral decisions to candidates by nomination. It is shown that if the credible platform commitments of the candidates is feasible, then at least one of the parties nominates in equilibrium to a candidate who has an ideology that is more radical than the delegating party's ideology. In a variety of circumstances, this, in turn, yields a polarization of equilibrium policy choices of the candidates. It is thus argued formally here that strategic nomination of the candidates may well be one of the major reasons behind the well documented observation that the platforms associated with the political parties in two-party democracies are often surprisingly polarized.

Keywords and Phrases: Delegation, Political competition, Polarization. JEL Classification Numbers: D72.

\footnotetext{
* We thank Alberto Alesina, Levent Koçkesen, Antonio Merlo, Ronny Razin, Vijay Krishna, Alessandro Lizzeri, and seminar participants at Alicante, Columbia, Copenhagen, and NYU for helpful comments. We also thank an anonymous referee for its useful suggestions. A good fraction of this research was conducted while Ok was a visitor in the Department of Economics at University of Alicante; he thanks for the kind hospitality of this institution. We gratefully acknowledge the financial support from the Spanish Ministry of Education through grant CICYT BEC2001-0535 (Faulí-Oller) and BEC20010980 (Ortuño-Ortín). 


\section{Introduction}

It is now widely recognized that in two-party political systems the proposed policies need not converge to the ideal policy of the median voter. If parties care not only about winning the elections but also about the implemented policies, and there is uncertainty about the distribution of voters, the equilibrium strategies may well dictate that each party propose a different policy. Some even argue that not only are the proposals different from the median voter's ideal policy, but they are also somewhat polarized. Indeed, a considerable fraction of the presidential candidates in the US are often claimed to be more radical than most members of their parties. ${ }^{1}$

There are several alternative explanations of polarized politics. In particular, in an influential set of works, Alesina and Rosenthal [1],[2],[3], advance an argument based on the incentives created by the "checks and balances" of the American political system. They take the view that due to these "checks and balances" the implemented policy is a compromise between the president and the Congress, and this, in turn, gives each party an incentive to choose a radical policy. Since the final policy will be in between the policies proposed by the parties (assuming that one party wins the presidency and the other the Congress), it is in the interest of a party to choose a radical policy to "move" the final policy in its desired direction. ${ }^{2}$

Of course, it makes sense to talk about more or less polarized proposals only when parties can make credible commitments to their proposals. The situation considered by Alesina and Rosenthal [1],[2], is one in which such commitment does not exist so that the proposal of a party coincides with its ideal policy. The "checks and balances" still moderate the final policy but through the strategic actions of the voters, not the parties. An interesting way to complement this sort of an investigation is, therefore, to consider the possibility that parties choose their candidates in a strategic way while the individuals vote sincerely. This opens up strategic possibilities for a party due to the fact that the ideal policies of the candidates of a party need not be identical to that of the party. Thus, in this scenario the polarization of party platforms in the elections may arise through strategic nomination of the candidates by the parties.

In this paper we examine precisely this scenario, and try to understand if and when polarization of platforms obtains through the route of strategic delegation to the radicals. Put more concretely, our objective is to understand if there is any room for political parties to nominate their candidates to influence the electoral strategy

1 Poole and Rosenthal [19] provides some empirical evidence showing that the political view of candidates is often more radical than the average one within their own parties. It appears that parties nominate radical candidates in actuality, who are then associated with radical policies. See Alesina and Rosenthal [1] for more on this.

2 There are of course other explanations. Feddersen [9] provides a costly voting model that explores the connection between voter participation and policy polarization. Baron [5] considers the effect of lobbying in a model where parties seek to maximize the probability of winning. He shows that in equilibrium policies will be polarized because parties have incentives to propose extreme policies in order to please the lobby group. Ortuño-Ortin [17] provides a simple model without legislature in which the implemented policy is a compromise between the two distinct policy proposals. In a similar vein with Alesina and Rosenthal [3], Ghosh [13] shows that the possibility of post-election bargaining may lead to pre-election policy extremism. Radical platforms can be also seen as the result of the greater weight extreme members have in the party's decision process. 
of the other party. This view sees the electoral process in itself as a mechanism resembling the "checks and balances," but one that operates prior to the elections.

We consider a largely standard model in which the policy space is unidimensional and there is uncertainty about the distribution of voters' preferences. There are two ideological parties who would like to see their respective ideal policies implemented, but they need to first obtain the approval of the majority of voters. Given the surrounding uncertainty, both parties aim to maximize their expected utility. In each party there is a set of potential ideological candidates which contains radicals and moderate types. A radical (moderate) candidate has a more (less) extreme ideal policy than the one associated with the party. In the first stage each party nominates (simultaneously) its candidate. In the second stage candidates make credible policy proposals. In the third stage voters vote and the winning candidate implements its announced proposal. Our main result states that, under fairly general assumptions, it cannot be the case that both parties delegate on moderate candidates in equilibrium: At least one of the candidates must be more radical than its party. ${ }^{3}$ In turn, the platform declarations of the nominated candidates may or may not be more polarized than the platforms without delegation. Thus the model is consistent with the often advanced view that the platforms adopted during the elections by otherwise radical candidates may well be quite close to each other. ${ }^{4}$ Interestingly, however, this situation obtains only when one of the nominated candidates is radical and the other is moderate. The model maintains that when both of the parties choose to nominate radical candidates, then the associated equilibrium platforms must be more polarized than the platforms without delegation.

The main thesis of this paper may then be stated succinctly as follows: To the extent that the nature of the political institutions maintain the feasibility of credible platform commitments of the candidates, the political parties would have strong strategic incentives to delegate to radical candidates. What is more, in such circumstances, the nominated candidates would instigate more polarized platforms than that would obtain in the absence of candidate delegation. ${ }^{5}$

As a passing remark, we note that our results are in concert with the empirical evidence on the ideological position of the 1876-1988 US presidential candidates reported in Alesina and Rosenthal [1] who compare the Poole and Rosenthal [18] estimates of the liberal-conservative position of US presidential candidates to the mean and one standard deviation position for the Senate. If we identify "radical" candidates as the ones with an ideological position outside the \pm one standard devi-

3 This conforms well with the factual observation made by Alesina and Rosenthal [1, p. 36]: "What is striking is not just that presidential candidates [who served in the Congress], including the succesful ones, were distant from the overall median [of the Congress] in many cases, but they often represented relatively extreme positions within their own parties."

${ }^{4}$ See Calvert [7] and Alesina and Rosenthal [3].

5 One key assumption here is that the true type of the candidates are observable by the voters. While this is surely a penetrating postulate, it appears quite realistic in the context of political competition where the identities of the potential candidates in the elections are almost always exposed to the public far before the actual nomination/primaries stage. Consequently, the usual arguments made against the assumption of observability of contracts in the literature on strategic delegation do not really apply to the present context. This, in our view, renders the investigation of the influence of strategic nomination of candidates on the electoral process even more relevant. 
ation band, then the election in 1900, with McKinley and Bryan as the candidates, is the only case in which both candidates are moderate. In the rest of the cases at least one (but often not both) of the candidates is found to be radical. ${ }^{6}$ It is important to notice that, as mentioned above, there are alternative models that can explain the existence of radical candidates. Most of these models, however, do not provide a satisfactory explanation of why we observe equilibria in which one candidate is radical and the other candidate is moderate. By contrast, a major advantage of the present model is its ability to sustain such interesting asymmetric equilibria.

\section{The political delegation game}

We consider a standard spatial competition model in which the policy space is the interval $[0,1]$ and there is a continuum of agents. The agents are assumed to have euclidean preferences on the policy space. Put precisely, following Alesina and Rosenthal [2], we represent the preference relation of agent $i \in[0,1]$ by a von Neumann-Morgenstern utility function $u_{i}:[0,1] \rightarrow \mathbf{R}$ given by

$$
u_{i}(z):=u(|z-i|),
$$

where $u:[0,1] \rightarrow \mathbf{R}$ is an arbitrary twice continuously differentiable function with $u^{\prime}<0$ on $(0,1], u^{\prime \prime}<0$ and $u^{\prime}(0)=0$. It is clear that this specification endows each agent with "single-peaked" preferences, and allows us to identify them with their ideal policies.

We posit that there are two political parties, $A$ and $B$, but depart from the standard Downsian model which envisages that the only motivation for the parties is the desire to win elections. Instead, we model the political parties here as "ideological" (or "policy-oriented") by treating each of them as an agent with preferences over the policy space (which may coincide, for instance, with that of the median or the average member of the party). ${ }^{7}$

As is almost exclusively done in the literature, and to distill the basic argument that we advance here to its simplest form, we ignore here the initial phase of the primaries. Thus, denoting the ideal policy of party $A$ by $z_{A}$, we view the party $A$ as possessing the utility function $u_{z_{A}}$. Similarly, party $B$ has the ideal point $z_{B}$ and the utility function $u_{z_{B}}$. Since we think of the party $A$ as the "left" party and $B$ as the "right" party, it is in the nature of things to assume that $0<z_{A}<z_{B}<1$.

In the standard one-stage formulation of the associated voting game, parties $A$ and $B$ simultaneously propose their policies, say, $x$ and $y$, respectively. Given the proposal profile $(x, y)$, each agent votes for the proposal he likes the most.

6 The Poole-Rosenthal estimates are available only for those candidates who served in the Congress. But as stated by Alesina and Rosenthal [1, p. 37], “...Even so, a casual observation of the candidates who had not served in the Congress would suggest that although Dewey, Eisenhower, and Carter were moderates, Stevenson, Dukakis, and Reagan were not. ... moderate, middle-of-the-road politicians are underrepresented as presidential candidates. ... The evidence is clear: even though, once selected, candidates must compete for the presidency in a general election, they fail to adopt convergent positions."

7 This alternative model is proposed and extensively studied in the seminal contributions of Wittman [24],[25] and Calvert [7], and is adopted in a good number of recent papers (cf. Alesina and Rosenthal [2],[3] and Roemer [20],[21]). 
Since agents have symmetric euclidean preferences, they vote for the one that is the closest to their ideal policies. The party that obtains more than half of the votes wins the election, and its proposed policy is then implemented. Ties are broken by a random draw (i.e., each party wins with probability $1 / 2$ in the case of a tie in votes). Given that agents have "single-peaked" preferences, $x$ is the winning proposal if $u_{m}(x)>u_{m}(y)$, where $m$ is the median agent in the society.

Clearly, the only relevant information about the distribution of voters here is the value of $m$. If both parties know this value, the assumption that parties are ideological loses its significance, and in accordance with the median voter theorem, both parties propose in equilibrium the same policy which coincides with the ideal point of the median agent. However, here we shall make the more realistic assumption that there is uncertainty about the distribution of voters, or more precisely, about the value of the median type $m$. Consequently, in what follows, we treat $m$ as a random variable the distribution of which is common knowledge for the involved parties. To simplify things at the onset, we shall assume initially that this random variable is distributed uniformly on $[0,1]$. As will be demonstrated in the sequel (see Sect. 3.2), this distributional assumption can be substantially weakened without altering the basic message of the paper.

If parties propose $(x, y)$, the average policy

$$
x \| y:=\frac{x+y}{2}
$$

acts as the cutoff point in that, while the agent with ideal policy $x \| y$ is indifferent between $x$ and $y$, any agent with type lower than $x \| y$ prefers $\min \{x, y\}$ over $\max \{x, y\}$. In this case, party $A$ wins the election if $x<y$ and $x \| y>m$, or if $x>y$ and $x \| y<m$, while each party wins with probability $1 / 2$ when $x \| y=m$. Thus, given that $m$ is uniformly distributed, the parties believe that, at the policy profile $(x, y)$, the probability that party $A$ will win the election is given by

$$
\pi(x, y):= \begin{cases}x \| y, & x<y \\ 1 / 2, & x=y \\ 1-x \| y, & x>y\end{cases}
$$

How does a party decide to propose a particular policy? Consider, for concreteness, party $A$. Since we have identified the ideology of this party with the preferences of an agent of type $z_{A}$, who perhaps is the median member of the party, it is somewhat reasonable to assume that this particular agent chooses $x$, the policy proposal. However, it might be in the interest of this agent to delegate this decision to a different agent, which will be referred to as a candidate for party $A$, in order to fare better in the elections against party $B$. While such a candidate is presumably a member of party $A$, she might nevertheless have an ideal policy different from $z_{A}$, and what is more, it is understood by everyone that this candidate herself will declare a policy proposal for the party. Thus, there is room for the party (acting as the delegator/principal) to strategize in its choice of a candidate (who will act as the delegatee/agent). In particular, it may choose to go for a radical candidate whose ideal policy $a$ lies to the left of $z_{A}$ (that is, $a<z_{A}$ ), or it may delegate to a 
moderate candidate with $a \in\left(z_{A}, z_{B}\right]$. (The case $a=z_{A}$ can be interpreted as no strategic delegation taking place.)

Before proceeding any further, let us reemphasize the way in which the delegation takes place in the present model. We assume here that candidates are chosen through nominations by the party leader or a committee of a few party members (as in some European countries such as Italy), and not through the primaries. While it will eventually be very interesting to augment the present model with a model of primaries, the absence of a standard model in this regard and the complexity of the voting game we consider here make this task hardly routine. ${ }^{8}$ To understand the basic effect of strategic delegation in the context of political competition, it seems reasonable at present to trivialize the primaries stage by considering candidates as "nominated by the party." While this admittedly falls short of providing a formal model of elections with primaries (a challenging objective in and of itself), it hopefully takes a step forward in this direction by providing a benchmark model of two-stage elections.

This formulation brings us very close to the literature on strategic delegation. It is then important to understand the information theoretic features of the present environment. After all, since Katz [14], it is well understood in this literature that the strategic delegation does not alter the equilibrium outcomes in simultaneous-move games if the contracts between the principals and the agents are not observable. ${ }^{9}$ On the other hand, if the contracts are observable by all parties, various credible commitment opportunities arise for the principals, and this yields a significant alteration of the equilibrium that would have obtained in the absence of delegation. It is important to note that, in contrast to firm theory, there is reason to view the latter assumption as more suitable in our context. Indeed, it is hardly unreasonable to assume that the preferences of the party and the preferences of any potential candidate are, in effect, observable by the public. In the real world, the political candidates are almost always well-known public figures whose reputations are well established prior to the nomination stage. Thus it is without loss of much realism to posit that the true types of the candidates are observable by all the concerned parties. This, in turn, makes strategic considerations viable, and as we shall show subsequently, has quite striking implications regarding the identity of the chosen candidates.

To summarize, then, we model the political competition as a two-stage extensive game with complete information which we refer to as the political delegation game. In the first stage of the game the parties name simultaneously their candidates. The set of potential candidates for party $A$ is that of all moderate and radical agents relative to the party ideal, i.e., $\left[0, z_{B}\right]$. Similarly, the action space of party $B$ is $\left[z_{A}, 1\right]$. In the second stage of the game, the candidates choose (again simultane-

${ }^{8}$ As noted by Alesina and Rosenthal [1, Sect. 2.7], the issues regarding primaries are quite complicated due to the facts that these often take place in different dates in different states. Furthermore, direct primary election laws differ significantly from state to state, and different systems are likely to have markedly different strategic implications. See, Gerber and Morton [12] for an overall view of the issues at hand.

9 This is not, however, necessarily the case when the original games played by the principals/parties have a sequential structure. See Fershtman and Kalai [11] and Koçkesen and Ok [15]. 
ously) their policy proposals, and the population vote sincerely over these policies knowing that the winning party will implement its announced proposal (the credible commitments scenario). As noted in the previous section, the question that we are interested in here is if it is beneficial for the parties to choose radical candidates to represent them in the elections.

To make things more precise, let us begin with analyzing the second stage game played by the chosen candidates. The expected utility for candidate $t$ at the proposal profile $(x, y)$ is given by the function $U_{t}:[0,1]^{2} \rightarrow \mathbf{R}$ as

$$
U_{t}(x, y):=\pi(x, y) u_{t}(x)+(1-\pi(x, y)) u_{t}(y), \quad t=a, b .
$$

Thus, the candidate game played between the candidates $a$ and $b$ is defined formally as

$$
G(a, b):=\left([0,1], U_{t}\right)_{t=a, b} .
$$

(This formulation is identical to the one suggested by, say, Wittman [25], and is largely standard.) A Nash equilibrium of $G(a, b)$ is called a candidate equilibrium, and the set of all such equilibria is denoted as $\mathcal{N}(a, b)$. A generic element of $\mathcal{N}(a, b)$ is in turn written as $(x(a, b), y(a, b))$.

Party $A$ can nominate any candidate in $\left[0, z_{B}\right]$ and party $B$ any candidate in $\left[z_{A}, 1\right]$. Take an arbitrary candidate profile $(a, b) \in\left[0, z_{B}\right] \times\left[z_{A}, 1\right]$, and consider an equilibrium profile $(x(a, b), y(a, b))$ in $\mathcal{N}(a, b)$. We define

$$
\Pi(a, b):=\pi(x(a, b), y(a, b))
$$

which stands for the probability that the candidate of party $A$ will win the elections if the path of play has it that $A$ chooses $a, B$ chooses $b$, and the equilibrium $(x(a, b), y(a, b))$ is played in the second stage of the game. In turn, the expected utility of party $T$ at this history is found as

$$
V_{T}(a, b):=\Pi(a, b) u_{z_{T}}(x(a, b))+(1-\Pi(a, b)) u_{z_{T}}(y(a, b)), T=A, B .
$$

We then say that $\left(a^{*}, b^{*}\right)$ is a party equilibrium, if

$$
\begin{aligned}
& V_{A}\left(a^{*}, b^{*}\right) \geq V_{A}\left(a, b^{*}\right) \text { and } \\
& V_{B}\left(a^{*}, b^{*}\right) \geq V_{B}\left(a^{*}, b\right) \text { for all }(a, b) \in\left[0, z_{B}\right] \times\left[z_{A}, 1\right] .
\end{aligned}
$$

Clearly, $\left(a^{*}, b^{*}\right)$ and $(x(a, b), y(a, b))_{(a, b) \in\left[0, z_{B}\right] \times\left[z_{A}, 1\right]}$ can together be thought of as a subgame perfect equilibrium of the political delegation game described above.

As for the rationality demands of the candidate and party equilibria described above, one major difficulty is the possibility of multiple candidate equilibria, which gives rise to the well known coordination problems. Moreover, this possibility complicates the subsequent analysis significantly. It will thus be useful here to introduce an assumption on the primitives of the model that will get rid of this difficulty. Fortunately, there is a somewhat natural way of doing this in our framework.

[A1]. $\frac{u^{\prime \prime}}{u^{\prime}}$ is strictly decreasing on $[0,1]$.

To understand the conceptual basis of this assumption, consider two candidates $a<a^{\prime}$ and two policies $x$ and $y$ with $a<a^{\prime}<x<y$. Now consider any 
lottery on the policies $x$ and $y$ in which both policies may be adopted with positive probability. By continuity of the preferences, there must exist a certainty equivalent of this lottery, say $z \in(x, y)$, for candidate $a^{\prime}$; that is, $a^{\prime}$ is indifferent between the said lottery on $\{x, y\}$ and the sure bet $z$. How about the candidate $a$ ? If [A1] holds, then this candidate is not indifferent between these lotteries; (s)he prefers the nondegenerate lottery to the sure bet $z$. So, in a clear sense, candidate $a$ is less afraid of losing the elections. That is, in the trade-off between probability of victory and its proposed policy, $a$ puts less weight on victory than candidate $a^{\prime}$. Therefore, $a$ will choose a platform closer to its ideal point even though this increases the probability of losing the election. ${ }^{10}$

The conceptual upshot here is that, given the platform of her competitor, a radical candidate is more "rigid" on her views so that she is more uncompromising than a more moderate candidate (in particular his/her own party). Interestingly, various versions of this view has already been advanced in the literature. For instance, the recent work of Blomberg and Harrington [6] focus on the beliefs of the agents, and show that radical members of the U.S. Congress tend to be rigid whereas moderates tend to be more flexible. This is precisely the implication of [A1] in the present context in which the focus is on the utility functions of agents as opposed to their beliefs.

Assumption [A1] turns out to be a very useful postulate for the present framework for, in addition to its appealing conceptual basis, it endows the model with a technical structure powerful enough to deliver a number of interesting formal results. Foremost, this property alone entails the existence and uniqueness of the candidate equilibrium.

Lemma 1. For any $0 \leq a<b \leq 1$, there exists a candidate equilibrium $\left(x^{*}, y^{*}\right) \in$ $\mathcal{N}(a, b)$, and we have $a<x^{*}<y^{*}<b$ for any such equilibrium. Furthermore, if [A1] holds, the candidate equilibrium of $G(a, b)$ is unique.

This fact shows that the candidate game $G(a, b)$ is quite well-behaved with regard to the existence and uniqueness of its equilibria. Unfortunately, the same cannot be said for the political delegation game in general. Indeed, this game is an infinite extensive game with imperfect information (due to the simultaneity of moves in the candidate game), and as is long known in game theory, it is quite difficult to find primitive conditions for such games that guarantee the existence of equilibria. Nevertheless, as we shall show below, it is still possible to identify the properties of arbitrary equilibria of our political delegation game, when at least one such equilibrium exists.

10 Notice that [A1] imposes that the absolute degree of risk aversion of the function $u$ is strictly increasing. While this may seem puzzling at first, we stress that $u$ is not defined on wealth as in most economic scenarios, where the opposite assumption is commonly maintained. 


\section{Main results}

\subsection{Two-sided radical delegation}

What will be the structure of equilibrium candidate nominations of each party in the political delegation game described above? Given the actions of others, nominating a moderate candidate will increase the chances of winning the election for a party, but perhaps at the cost of having to live with an implemented policy that is too far from the party ideal. On the other hand, a radical candidate may prove to be a poor competitor in the elections. Clearly, there is a trade off between increasing the probability of winning and implementing a relatively desirable policy for each party. Thus, a priori, it is not at all obvious if there exists a clear-cut prediction about the structure of equilibrium nominations. Curiously, however, in the present setting a very concrete answer is available:

Theorem 1. If [A1] holds, then $a^{*}<z_{A}$ and $b^{*}>z_{B}$ for any party equilibrium $\left(a^{*}, b^{*}\right)$, that is, both parties delegate in equilibrium to radical candidates.

The reasoning behind this result becomes relatively transparent once one recognizes that the game played by any two distinct candidates in the second stage of the political delegation game is actually supermodular. Thus there is a close relation between Theorem 1 and the strategic delegation results obtained by Fershtman and Judd [10] and Sklivas [22] in the case of a Bertrand duopoly with differentiated outputs. $^{11}$

While its formal proof is included in the Appendix, it may be a good idea to provide some intuition for Theorem 1 at this point. Consider the situation in which each party has nominated a candidate whose ideal point is identical to that of the corresponding party. Why is it not possible for this to be a party equilibrium? The key observation is that party A knows that the candidate $z_{B}$ has an increasing reaction function. (This is because when her opponent proposes policies further and further away from $z_{B}$, the convexity of the preferences of candidate $z_{B}$ forces her to view the increase in the probability of victory less pressing than the misery that he will sustain in case of a loss, thereby pushing her to propose policies further and further away from his own ideal to increase further the probability of victory.) Consequently, given that in elections it will face a candidate of type $z_{B}$, party $A$ has an incentive to delegate to at least a slightly more radical candidate $a \in\left[0, z_{A}\right)$. Such a candidate may do slightly worse than the party itself in the elections with respect to the probability of winning. But, due to the concavity of the party preferences, this negative effect is dominated by the positive effect of forcing the other party to propose a policy that is closer to $z_{A}$ than the previous one (which is presumably quite far away from $z_{A}$ ). Of course, party $B$ reasons the same way, and hence the best response dynamics maintain that, starting from the candidate profile $\left(z_{A}, z_{B}\right)$,

11 We should note, however, that the technical structure of our model is more complicated than the duopoly model analyzed by these authors who have assumed linear demand and cost structures (and even than the generalization provided by Koçkesen, Ok and Sethi [16] who relaxed these linearity assumptions). Indeed, in contrast with the present case, obtaining the analogue of Lemma 1 for linear Bertrand duopoly with differentiated products is an easy exercise. 


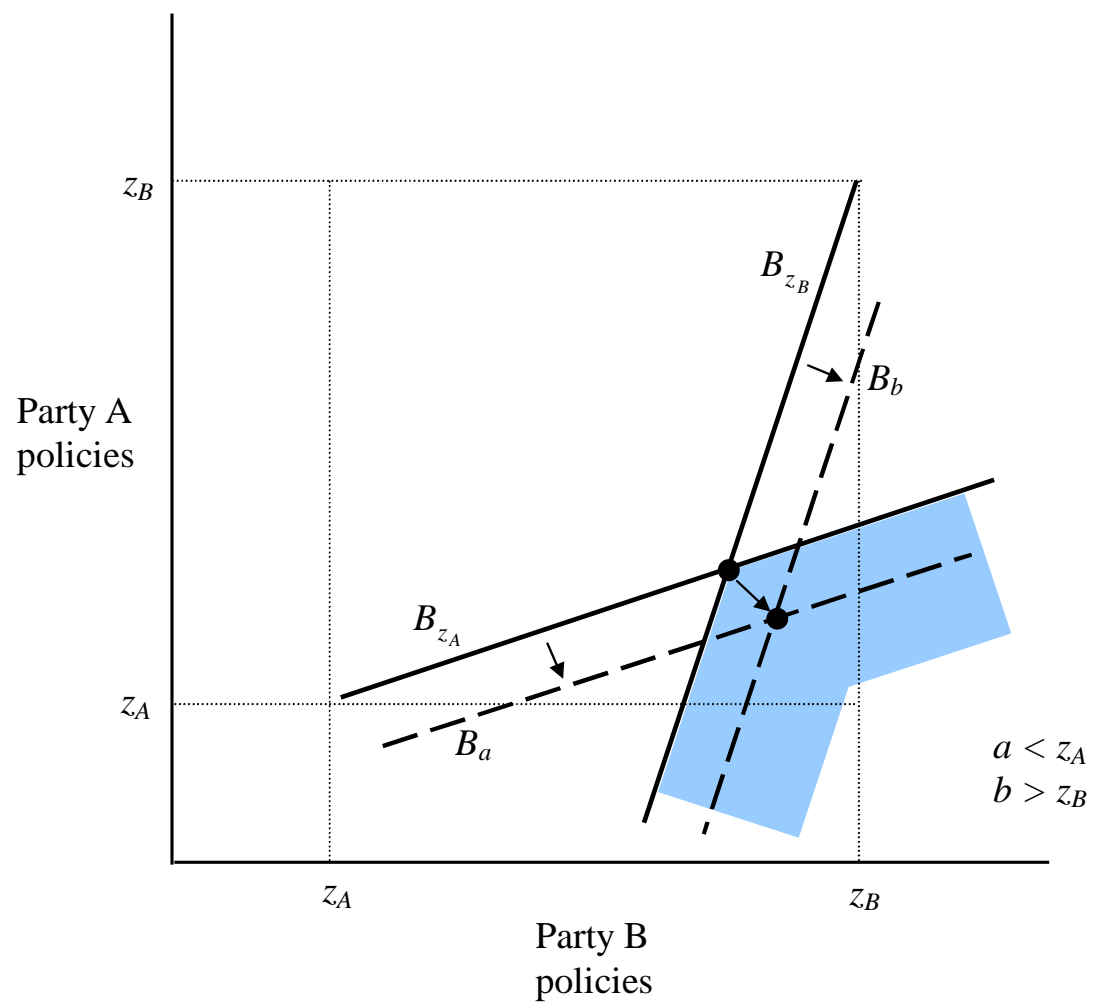

Figure 1

one is to move to a more radical profile (see Figure 1). If an equilibrium exists, this must be one in which both parties nominate a radical candidate. ${ }^{12}$

While it is interesting in its own right, Theorem 1 falls short of providing a prediction regarding the polarization of platforms chosen by the candidates in equilibrium. In principle, it could happen that our radical candidates propose platforms that are less polarized than the platforms that would obtain in the absence of delegation. In fact, it is possible that a radical candidate plays the voting game more moderately than her own party in equilibrium. ${ }^{13}$ However, it turns out that the joint play of radical candidates is bound to yield a more polarized pair of equilibrium platforms relative to that induced by the play of the principal parties. To formalize this point, let us agree to refer to a pair of platforms $(x, y)$ as more polarized than

12 An interesting extension of the model obtains by allowing the nominations to take place sequentially. In this case, a corollary of the present analysis has it that the party who nominates its candidate after the other party has nominated its own will delegate to a radical candidate in equilibrium. (The proof is contained within the proof of Theorem 1.)

13 That is, $\mathcal{N}(a, b)$ need not be monotonic in $a$ and $b$, a fact which is typical of Hotelling-type games. A concrete example of a class of candidate games (with the ideal points of the society being distributed according to a Beta law) that possess such non-monotonic equilibrium sets will be provided in Section 4 . 
the pair of platforms $\left(x^{\prime}, y^{\prime}\right)$ whenever $|y-x|>\left|y^{\prime}-x^{\prime}\right|$. As a nontrivial implication of Theorem 1, we may then obtain the following result on the polarization of equilibrium platforms in the present model.

Theorem 2. Let [A1] hold, and let $\left(a^{*}, b^{*}\right)$ be a party equilibrium. If $\left(x^{*}, y^{*}\right) \in$ $\mathcal{N}\left(a^{*}, b^{*}\right)$ and $\left(x^{\prime}, y^{\prime}\right) \in \mathcal{N}\left(z_{A}, z_{B}\right)$, then the platform $\left(x^{*}, y^{*}\right)$ is more polarized than the platform $\left(x^{\prime}, y^{\prime}\right)$.

That is, in equilibrium, the (unique) pair of platforms chosen by radical candidates is more polarized than the (unique) pair of platforms that would obtain had the parties did not at all engage in delegation activities and instead played the game themselves. Thus, in the present model, strategic nomination of the candidates yields not only the polarization of the "types" of the running candidates, but also that of the equilibrium platforms.

It is possible to illustrate Theorem 2 as well by using Figure 1 . The key observation here (which requires formal proof) is that the slope of the reaction functions of any radical candidate is lower than one. Moreover, a simple graphical argument shows that any point $(x, y)$ within the shaded area satisfies that $y-x>y^{\prime}-x^{\prime}$. But by Theorem 1 both of the candidates are radical and the equilibrium platforms must be in the shaded area, and hence Theorem 2. A rigorous proof that formalizes these steps is provided in the Appendix.

Finally, what of a welfare analysis? While the conclusions of the model are ambiguous in most cases, Theorem 2 allows us derive a clear prediction in the case where the ideal points of the parties are equally apart from the midpoint $1 / 2$ of the political continuum. In this case the expected policy without delegation coincides with the expected policy with delegation. But Theorem 2 says that polarization of platforms is greater with delegation, so the lottery (over platforms) in the absence of delegation second order stochastically dominates that obtains with delegation. Consequently, all voters (who have risk averse preferences) are found to be better off without delegation in this particular case.

\subsection{One-sided radical delegation}

Theorems 1 and 2 suffer from two serious shortcomings. First of all, they use crucially an unacceptably strong uniformity assumption regarding the distribution of the median type in the population. Secondly, the conclusion of Theorem 1 is somewhat "too strong" in the sense that empirical studies, as well as introspection, indicate that it is not all that common that both the republicans and the democrats in the US Presidential elections nominate radical candidates. Consequently, both the theoretical reach and the predictive power of the model would be strengthened if we could generalize the present analysis to cover a large class of distributions, and as a result, "weaken" the conclusion of Theorem 1 from "both candidates are radicals" to "at least one candidate is radical." Our objective in this section is to do precisely this.

In what follows, we consider the political delegation model introduced in Section 2 with the distribution of the median type being given by an arbitrary absolutely 
continuous distribution function $F:[0,1] \rightarrow[0,1]$ with a strictly positive density $f:[0,1] \rightarrow \mathbf{R}_{++}$. Consequently, the only modification of the formal model is through changing the definition of the probability of winning function $\pi$ to

$$
\pi(x, y):= \begin{cases}F(x \| y), & x<y \\ 1 / 2, & x=y \\ 1-F(x \| y), & x>y\end{cases}
$$

at the policy profile $(x, y)$. In what follows, the only regularity assumption we impose on the distribution function $F$ is a commonly used logconcavity postulate.

[A2]. Both $F$ and $1-F$ are logconcave functions.

It is well known that the most commonly used distribution functions satisfy this assumption. ${ }^{14}$ In particular, the uniform distribution satisfies [A2], and hence the present model is indeed a generalization of the one we considered in Sections 2 and 3.1 .

It turns out that, under assumptions [A1] and [A2], the exact analogue of Lemma 1 holds true. In fact, the assumptions [A1] and [A2] are actually all that one needs to turn Theorem 1 to a one-sided radical delegation result. The following is, then, our main finding:

Theorem 3. If [A1] and [A2] hold, then either $a^{*}<z_{A}$ or $b^{*}>z_{B}$ for any party equilibrium $\left(a^{*}, b^{*}\right)$, that is, at least one party delegates in equilibrium to a radical candidate.

We do not obtain two-sided radical delegation in general because one cannot exclude the possibility that the slope of the reaction function of one of the candidates is downward sloping if $\left|f^{\prime}(x \| y)\right|$ is high enough. In this case, the strategic effect of delegation of moving the policy of the competing candidate closer to the ideal point of the party is obtained by delegating to moderate candidates. ${ }^{15}$ Indeed, as we shall show in Section 4, it is impossible to tighten Theorem 3 to a two-sided delegation result. Nevertheless, Theorem 3 shows that it is still impossible to have both candidates moderate. Consequently, the model at hand, in general, allows for asymmetric equilibria in which one candidate is radical while the other is moderate (which is in concert with the empirical evidence reported in Alesina and Rosenthal [1] for the U.S. presidential elections).

At this level of generality, it is not possible to say much about the polarization of the equilibrium platforms when only one of the candidates is radical. But depending on the precise structure of the distribution of the median type in the society, of course, it is quite possible that both candidates are radical. In this case, the geometric logic behind Theorem 2 kicks in, and we obtain a straightforward counterpart of this result in the present (general) setup.

14 This assumption is weaker than log-concavity of the density function $f$; see Barlow and Proschan [4]. The normal, Laplace, gamma, exponential, beta (with restrictions on the parameters), Weibull and Dirichlet are examples of logconcave distributions, see Caplin and Nalebuff [8]. An alternative (stronger) assumption would be quasi-concavity of $f$. This is adopted in, for example, Weber [23].

15 This result is akin to the one in Fershtman and Judd [10] and Sklivas [22] where the chosen type of delegation depends crucially on whether firms compete in prices (upward-sloping reaction functions) or in quantities (downward-sloping reaction functions). 
Theorem 4. Let [A1] and [A2] hold, and let $\left(a^{*}, b^{*}\right)$ be a party equilibrium with $a^{*}<z_{A}$ and $b^{*}>z_{B}$. If $\left(x^{*}, y^{*}\right) \in \mathcal{N}\left(a^{*}, b^{*}\right)$ and $\left(x^{\prime}, y^{\prime}\right) \in \mathcal{N}\left(z_{A}, z_{B}\right)$, then the platform $\left(x^{*}, y^{*}\right)$ is more polarized than the platform $\left(x^{\prime}, y^{\prime}\right)$.

The welfare implications of this result is identical to the uniform case, provided that the distribution associated with $F$ is symmetric and the ideal points of the parties are equally apart about the midpoint $1 / 2$. In this case the expected policy without delegation coincides with the expected policy with delegation, but due to Theorem 4, all risk averse voters are better off without delegation.

\section{Numerical examples}

Due to its formal generality, there are a number of issues that we have left unaddressed in the analysis so far. First of all, at the moment we do not have a robust example for which a party equilibrium exists. Second, it is not at present clear if Theorem 3 can be tightened to a two-sided delegation result (as it is the case in Theorem 1). Third, we do not yet know if the radicality of a candidate necessarily translates into the radicality of her optimal platform choice in the elections. Finally, little is said so far about the polarization of equilibrium platforms when there is a only one radical candidate in the political competition. In this section, we shall address each of these issues by means of a numerical example. ${ }^{16}$

We consider an example in which the ideal policy for party $A$ is $z_{A}=0.25$ and the ideal policy for party $B$ is $z_{B}=0.935$. The utility function of the agents is $u_{i}(z):=-|z-i|^{1.2}$ which clearly satisfies [A1]. The median voter is distributed according to the Beta distribution with parameters $(c, d)$; we denote the associated distribution function by $F(\cdot ; c, d)$. In what follows we fix $d=2$, and compute the party and candidate equilibria for a sequence of (two hundred) economies with different values of the parameter $c$, starting with $c=2.2$ and increasing up to $c=14$. In all of these economies the distribution function $F(\cdot ; c, 2)$ satisfies assumption [A2]. Moreover, higher values of the parameter $c$ can be thought of as associated with a more conservative electorate ${ }^{17}$. More precisely, one can show that, for all the values of the parameter $c$ considered in this exercise, if $c>c^{\prime}$ then the distribution $F(\cdot ; c, 2)$ first order stochastically dominates $F\left(\cdot ; c^{\prime}, 2\right)$.

In all of the economies considered here a unique equilibrium exists. The results for three selected economies are provided in Table 1 where $a^{*}$ and $b^{*}$ are the values of the candidates in the party equilibrium, and $x^{*}$ and $y^{*}$ are the equilibrium platforms. We also report the equilibrium platforms $x^{\prime}$ and $y^{\prime}$ that obtain when parties cannot delegate. Finally, the last column of Table 1 provides the probability of victory for party $A$.

Notice that for the value of $c=8$ the candidate of party $A$ is moderate $(0.25=$ $z_{A}<a^{*}=0.2820$ ). This shows that Theorem 3 cannot be tightened to a two-sided

16 The numerical computations were done using Mathematica, and the program is available from authors upon request.

17 If we set $c=d>2$, increases of $c$ can be seen as associated to a more concentrated electorate around $1 / 2$, i.e. a less polarized distribution of voters. One can easily show that a lower degree of such polarization doesn't not always imply a less polarized choice of candidates. 
Table 1

\begin{tabular}{cccccccc}
\hline$c$ & $a^{*}$ & $b^{*}$ & $x^{*}$ & $y^{*}$ & $x^{\prime}$ & $y^{\prime}$ & $\pi$ \\
\hline 3.15 & 0.2311 & 0.9986 & 0.3904 & 0.8594 & 0.3908 & 0.8470 & 0.49 \\
8 & 0.2820 & 0.9433 & 0.6595 & 0.9259 & 0.6568 & 0.9213 & 0.414 \\
12.95 & 0.2363 & 0.9362 & 0.7574 & 0.9338 & 0.7569 & 0.9327 & 0.341 \\
\hline
\end{tabular}

delegation result: depending on the distributional specification of the model, there may exist equilibria with only one candidate being radical.

It is also interesting to compare the platforms under the party equilibrium with the platforms in the no delegation case. The example at hand demonstrates that the candidate platforms do not necessarily monotonically depend on one's ideal point. For the value $c=12.95$, we have $a^{*}<z_{A}$ (the candidate is more radical than the party) and yet $x^{\prime}<x^{*}$ (the candidate proposes in equilibrium a less radical platform than the one proposed by her party in the no delegation case). However, not only is $\left(x^{*}, y^{*}\right)$ more polarized than $\left(x^{\prime}, y^{\prime}\right)$ for $c \in\{3.15,12.95\}$ where both candidates are radical (this is entailed by Theorem 4), but the same holds for $c=8$ where there is only one radical candidate (a case which is not covered by Theorem 4). Thus, the example at hand suggests that our theoretical results on the polarization of platforms provided in Section 3.2 might not depend on the two-sided radical delegation result.

The questions raised in the opening paragraph of this section are thus answered. The final order of business concerns the robustness of the numerical findings reported here. In this regard we note that the present results are robust to changes in the parameters and even in the choice of the distribution function. For instance, we can generate similar results for the model with $u_{i}(z):=-|z-i|^{\alpha}$ and $F(z)=z^{\beta}$ for a continuum of different values of the ideal points $z_{A}$ and $z_{B}$, and parameters $\alpha>1$ and $\beta \geq 1$.

\section{Conclusion}

In this paper we have attempted to show that the feasibility of credible platform commitments of the political candidates gives strong incentives to parties for choosing their political leaders from the pool of relatively radical candidates. Under a large set of circumstances, this, in turn, forces the political platforms declared by the candidates in the elections to be more polarized than that would obtain in the absence of delegation. This suggests that one of the major sources of the observed nonconvergence of platforms in the real-world two-party political systems is the very nature of the associated voting games that renders issues related to strategic delegation duly relevant.

There are two major limitations of the present work which must be addressed in future research. First of all, our entire analysis is confined to two-party elections. Unfortunately, the required extension to more than two parties is by no means routine since this requires a theory of government coalition formation. Perhaps a 
more pressing shortcoming is the silence of our approach with regard to the way decisions are taken within a political party, that is, the trivial way we have modeled the stage of primaries here. The absence of a standard game theoretical model of primaries attests to the fact that this is an entirely nonroutine exercise, but one which is to be necessarily taken up in the future.

\section{Appendix}

Proof of Lemma 1. The existence of an equilibrium $\left(x^{*}, y^{*}\right)$ is obtained by a direct application of Corollary 3.1 of Roemer [20]. To establish the rest of the assertions in Lemma 1, let us first observe that $u_{a}$ and $u_{b}$ are $C^{2}$ functions. That $u_{a}$ is $C^{2}$ on $[0, a) \cup(a, 1]$ follows readily from the associated differentiability properties of $u$. Moreover, we have $\mathbf{D}_{-} u_{a}(a)=-u^{\prime}(0)=0=u^{\prime}(0)=\mathbf{D}_{+} u_{a}(a)$ and $\mathbf{D}_{-} u_{a}^{\prime}(a)=u^{\prime \prime}(0)=\mathbf{D}_{+} u_{a}^{\prime}(a)$ so that $u_{a}$ is $C^{2}$ on the entire $[0,1]$ with

$$
u_{a}^{\prime}(x)=\left\{\begin{array}{r}
u^{\prime}(x-a), x \geq a \\
-u^{\prime}(a-x), x<a
\end{array} \quad \text { and } \quad u_{a}^{\prime \prime}(x)=\left\{\begin{array}{l}
u^{\prime \prime}(x-a), x \geq a \\
u^{\prime \prime}(a-x), x<a
\end{array} .\right.\right.
$$

An analogous reasoning applies also to $u_{b}$. These findings also show that $U_{a}$ and $U_{b}$ are $C^{2}$ everywhere on $[0,1]^{2}$ but the diagonal $\{(x, x): 0 \leq x \leq 1\}$.

Let $B_{t}:[0,1] \rightarrow[0,1]$ stand for the best response correspondence of agent $t=a, b$. An immediate application of Weierstrass' theorem shows that $B_{t}$ is well defined. We now examine the structure of the map $B_{a}$ a little further; similar observations apply also to the map $B_{b}$. Since $U_{a}(a, a)=u(0)$ is the unique maximum payoff for player $a$ in the game $G(a, b)$, we clearly have $B_{a}(a)=a$. Now take any $y \neq a$ and $x \in B_{a}(y)$. It is easy to see that $|a-x| \leq|a-y|$ (for playing $y$ for candidate $a$ is a better response to $y$ than playing any other $x$ with $|a-x|>|a-y|)$, and hence $u_{a}(x) \geq u_{a}(y)$. But then, if $a>x$ was the case, we would have

$$
\partial_{1} U_{a}(x, y)=\frac{1}{2}\left(u_{a}(x)-u_{a}(y)\right)-(x \| y) u_{a}^{\prime}(x)>0,
$$

which contradicts that $x \in B_{a}(y)$. Since $\partial_{1} U_{a}(a, y)=\frac{1}{2}\left(u(0)-u_{a}(y)\right)>0$, we must actually have $x>a$. Moreover, either $x>y>a$ or $x \geq 2 a-y>a$ implies

$$
\partial_{1} U_{a}(x, y)=\frac{1}{2}\left(u_{a}(x)-u_{a}(y)\right)+(x \| y) u^{\prime}(x-a)<0,
$$

disqualifying these ranges for $x$ and $y$. Finally, since $U_{a}(y, y)=u_{a}(y), x=y>a$ cannot hold for any $x \in B_{a}(y)$ either. In summary, we have

$$
x\left\{\begin{array}{ll}
\in(a, y) & y>a \\
=a, & y=a \\
\in(a, 2 a-y) & y<a
\end{array} \quad \text { for all } x \in B_{a}(y) .\right.
$$

Reasoning similarly for $B_{b}$, then, we may conclude that $a<x^{*}<y^{*}<b$, as we sought. Another implication of (3) is that

$$
u_{a}(x)>u_{a}(y) \quad \text { and } \quad u_{a}^{\prime}(x)>u_{a}^{\prime}(y) \quad \text { for all } x \in B_{a}(y), y \neq a
$$


since both $u$ and $u^{\prime}$ are strictly decreasing functions.

We next show that $B_{a}$ is a single-valued correspondence. To see this, take any $y \neq a$ and $x^{\prime}, x^{\prime \prime} \in B_{a}(y)$. Given (3), we must have $\partial_{1} U_{a}\left(x^{\prime}, y\right)=\partial_{1} U_{a}\left(x^{\prime \prime}, y\right)=$ 0 . But unless $x^{\prime}=x^{\prime \prime}$ this is impossible, because

$$
\partial_{11} U_{a}(x, y)=u_{a}^{\prime}(x)+(x \| y) u_{a}^{\prime \prime}(x)<0, \quad a \neq y \neq x \geq a,
$$

that is, $\partial_{1} U_{a}(\cdot, y)$ is a strictly decreasing function on $[0,1] \backslash\{y\}$. Thus, we may conclude that $B_{a}$ (and similarly, $B_{b}$ ) can be regarded as a function. (With a slight abuse of notation, then, we shall view $B_{a}(y)$ and $B_{b}(x)$ as members of $[0,1]$ in what follows.) Moreover, since interiority of the associated maximization problems are readily verified, we may use the first order conditions to write, for any $x, y \in[0,1]$,

$$
\frac{1}{2}\left[u_{a}\left(B_{a}(y)\right)-u_{a}(y)\right]+\left(B_{a}(y) \| y\right) u_{a}^{\prime}\left(B_{a}(y)\right)=0
$$

and

$$
\frac{1}{2}\left[u_{b}(x)-u_{b}\left(B_{b}(x)\right)\right]-\left(1-\left(x \| B_{b}(x)\right)\right) u_{b}^{\prime}\left(B_{b}(x)\right)=0
$$

which characterize the functions $B_{a}$ and $B_{b}$. By a standard argument based on the implicit function theorem, we may further deduce that both $B_{a}$ and $B_{b}$ are continuously differentiable functions. Implicitly differentiating (5) and rearranging, therefore, we obtain

$$
B_{a}^{\prime}(y)=\frac{u_{a}^{\prime}(y)-u_{a}^{\prime}\left(B_{a}(y)\right)}{2 u_{a}^{\prime}\left(B_{a}(y)\right)+\left(B_{a}(y)+y\right) u_{a}^{\prime \prime}\left(B_{a}(y)\right)} .
$$

By (4), we have $B_{a}^{\prime}(y)>0$ whenever $y \neq a$, whereas $B_{a}^{\prime}(a)=0$. We next claim that

$$
B_{a}^{\prime}(y)<1 \quad \text { whenever } \quad y>a .
$$

To see this, observe that $B_{a}^{\prime}(y)<1$ holds if and only if $u_{a}^{\prime}(y)-u_{a}^{\prime}\left(B_{a}(y)\right)>$ $2 u_{a}^{\prime}\left(B_{a}(y)\right)+\left(B_{a}(y)+y\right) u_{a}^{\prime \prime}\left(B_{a}(y)\right)$ which is implied by

$$
u_{a}^{\prime}(y)-u_{a}^{\prime}\left(B_{a}(y)\right)>\left(B_{a}(y)+y\right) u_{a}^{\prime \prime}\left(B_{a}(y)\right) .
$$

By (5), this last inequality can be rewritten as

$$
\left(u_{a}^{\prime}(y)-u_{a}^{\prime}\left(B_{a}(y)\right)\right) u_{a}^{\prime}\left(B_{a}(y)\right)-u_{a}^{\prime \prime}\left(B_{a}(y)\right)\left(u_{a}(y)-u_{a}\left(B_{a}(y)\right)\right)<0 .
$$

In turn, since $y>B_{a}(y)>a,(9)$ can be written as

$$
\begin{gathered}
\left(u^{\prime}(y-a)-u^{\prime}\left(B_{a}(y)-a\right)\right) u^{\prime}\left(B_{a}(y)-a\right) \\
-u^{\prime \prime}\left(B_{a}(y)-a\right)\left(u(y-a)-u\left(B_{a}(y)-a\right)\right)<0 .
\end{gathered}
$$

Now define the differentiable function $H:[0,1] \times(a, 1] \rightarrow \mathbf{R}$ by

$$
\begin{aligned}
H(v, k):= & {\left[u^{\prime}(v-a)-u^{\prime}(k-a)\right] u^{\prime}(k-a) } \\
& -u^{\prime \prime}(k-a)(u(v-a)-u(k-a)) .
\end{aligned}
$$

Clearly, $H(k, k)=0$. Moreover,

$$
H_{1}(v, k)=u^{\prime}(k-a) u^{\prime}(v-a)\left[-\frac{u^{\prime \prime}(k-a)}{u^{\prime}(k-a)}+\frac{u^{\prime \prime}(v-a)}{u^{\prime}(v-a)}\right],
$$


so, by [A1], we have $H_{1}(v, k)<0$ whenever $v>k$. It follows that $H(v, k)<0$ whenever $v>k$. Then, since $y>B_{a}(y)>a$, we find $H\left(y, B_{a}(y)\right)<0$ which is nothing but what is claimed in (10). As noted earlier, this, in turn, proves (7). An analogous observation also holds for $B_{b}$.

The uniqueness of equilibrium is now proved by a straightforward contraction argument. Let $\left(x^{*}, y^{*}\right)$ and $\left(x^{* *}, y^{* *}\right)$ both belong to $\mathcal{N}(a, b)$. Then we must have $\left(x^{*}, y^{*}\right)=B\left(x^{*}, y^{*}\right)$ and $\left(x^{* *}, y^{* *}\right)=B\left(x^{* *}, y^{* *}\right)$ where $B:[0,1]^{2} \rightarrow[0,1]^{2}$ is defined by $B(x, y)=\left(B_{a}(y), B_{b}(x)\right)$. But then, since $y^{*}, y^{* *}>a$ must hold (as was established earlier), by using (7) and the analogous finding for $B_{b}$, we observe that $\left(x^{*}, y^{*}\right) \neq\left(x^{* *}, y^{* *}\right)$ yields the contradiction:

$$
\begin{aligned}
\left\|B\left(x^{*}, y^{*}\right)-B\left(x^{* *}, y^{* *}\right)\right\|_{1} & =\left|B_{a}\left(y^{*}\right)-B_{a}\left(y^{* *}\right)\right|+\left|B_{b}\left(x^{*}\right)-B_{b}\left(x^{* *}\right)\right| \\
& <\left|y^{*}-y^{* *}\right|+\left|x^{*}-x^{* *}\right| \\
& =\left\|\left(x^{*}, y^{*}\right)-\left(x^{* *}, y^{* *}\right)\right\|_{1} \\
& =\left\|B\left(x^{*}, y^{*}\right)-B\left(x^{* *}, y^{* *}\right)\right\|_{1} .
\end{aligned}
$$

Proof of Theorem 1. Consider first the case in which $b^{*}=z_{A}<z_{B}$. In this case, $a^{*}$, being the best response of party $A$, must equal $z_{A}$. But then delegating to $z_{B}$ (instead of to $\left.b^{*}\right)$ is a better response for party $B$. Consequently, we have $z_{A}<b^{*}$. One can similarly check that $a^{*}<z_{B}$. Moreover, $z_{A}<b^{*} \leq a^{*}<z_{B}$ cannot hold, for in this case delegating to $a^{*}$ is a better response for party $B$. Consequently, we are left with two possibilities: $z_{A} \leq a^{*}<b^{*}$ and $a^{*}<z_{A}<b^{*}$. In what follows our objective is to eliminate the possibility of the former case. To this end, define the nonempty interval $J:=\left[z_{A}, \min \left\{b^{*}, z_{B}\right\}\right)$, and let $x(a):=x\left(a, b^{*}\right)$ and $y(a):=y\left(a, b^{*}\right)$ for all $a \in J$, where $\left(x\left(a, b^{*}\right), y\left(a, b^{*}\right)\right)$ is the unique equilibrium in $\mathcal{N}\left(a, b^{*}\right)$. By Lemma 1 we know that $x\left(a, b^{*}\right)<y\left(a, b^{*}\right)$, and hence, provided that $a<b^{*}$, both $U_{a}$ and $U_{b^{*}}$ are $C^{2}$ around an open neighborhood of $\left(a, b^{*}\right)$. Thus, the following first order conditions must be satisfied:

$$
\partial_{1} U_{a}(x(a), y(a))=\frac{1}{2}\left[u_{a}(x(a))-u_{a}(y(a))\right]+(x(a) \| y(a)) u_{a}^{\prime}(x(a))=0
$$

and

$$
\begin{aligned}
\partial_{2} U_{b^{*}}(x(a), y(a))= & \frac{1}{2}\left[u_{b^{*}}(x(a))-u_{b^{*}}(y(a))\right] \\
& -(1-(x(a) \| y(a))) u_{b^{*}}^{\prime}(y(a))=0 .
\end{aligned}
$$

Applying the implicit function theorem, we observe that both $x$ and $y$ are differentiable on the interior of $J$ (with left derivatives at $z_{A}$ ).

Now assume that $z_{A} \leq a^{*}<b^{*}$. To derive a contradiction, we shall show below that, for any fixed $a \in J$,

$$
\partial_{1} V_{A}\left(a, b^{*}\right)=\partial_{1} U_{z_{A}}(x(a), y(a)) x^{\prime}(a)+\partial_{2} U_{z_{A}}(x(a), y(a)) y^{\prime}(a)<0 .
$$

To this end, we begin by observing that, for each $z_{A} \leq x<y<1$,

$$
\begin{aligned}
\partial_{2} U_{z_{A}}(x, y) & =\frac{1}{2}\left(u_{z_{A}}(x)-u_{z_{A}}(y)\right)+(1-(x \| y)) u_{z_{A}}^{\prime}(y) \\
& <\frac{1}{2} u_{z_{A}}^{\prime}(y)(x-y)+(1-(x \| y)) u_{z_{A}}^{\prime}(y) \\
& =u_{z_{A}}^{\prime}(y)(1-y)<0
\end{aligned}
$$


where the first inequality follows from the fact that $u_{z_{A}}$ is a strictly decreasing and concave function on $\left[z_{A}, 1\right]$. Secondly, we differentiate (11) and (12) to obtain the system

$$
\left[\begin{array}{cc}
\partial_{11} U_{a}(x(a), y(a)) & \partial_{12} U_{a}(x(a), y(a)) \\
\partial_{21} U_{b^{*}}(x(a), y(a)) & \partial_{22} U_{b^{*}}(x(a), y(a))
\end{array}\right]\left[\begin{array}{l}
x^{\prime}(a) \\
y^{\prime}(a)
\end{array}\right]=\left[\begin{array}{c}
-\vartheta \\
0
\end{array}\right]
$$

where

$$
\begin{aligned}
\vartheta & :=\left.\frac{\partial}{\partial a} \partial_{1} U_{a}(x, y)\right|_{(x, y)=(x(a), y(a))} \\
& =\frac{1}{2}\left(u_{a}^{\prime}(y(a))-u_{a}^{\prime}(x(a))-(x(a)+y(a)) u_{a}^{\prime \prime}(x(a))\right) .
\end{aligned}
$$

By using the analogous reasoning that yielded (8) earlier, we find that $\vartheta>0$. Moreover, it is routine to check that the discriminant $\Delta$ of the linear system (14) is positive. Thus, since

$$
\begin{aligned}
x^{\prime}(a) & =\left(\frac{-\vartheta}{\Delta}\right) \partial_{22} U_{b^{*}}(x(a), y(a)) \\
& =\left(\frac{-\vartheta}{\Delta}\right)\left(u_{b^{*}}^{\prime}(y(a))+(1-x(a) \| y(a)) u_{b^{*}}^{\prime \prime}(y(a))\right)
\end{aligned}
$$

we find that $x^{\prime}(a)>0$. It is apparent from the second equation of (14) that $\operatorname{sgn}\left(x^{\prime}(a)\right)=\operatorname{sgn}\left(y^{\prime}(a)\right)$ so that we may also conclude that $y^{\prime}(a)>0$. Consequently, claim (13) will follow if we can show that

$$
\partial_{1} U_{z_{A}}(x(a), y(a))<0 .
$$

To this end, observe that

$$
\begin{aligned}
\frac{\partial}{\partial c} \partial_{1} U_{c}(x, y)= & \frac{1}{2}\left(u_{c}^{\prime}(y)-u_{c}^{\prime}(x)-(x+y) u_{c}^{\prime \prime}(x)\right)>0 \\
& \text { whenever } z_{A} \leq c<x<y
\end{aligned}
$$

which is again proved in a way analogous to (8). Consequently, letting $x=x(a)$ and $y=y(a)$, we get

$$
\frac{\partial}{\partial c} \partial_{1} U_{c}(x(a), y(a))>0 \quad \text { for all } z_{A} \leq c \leq a .
$$

But since $\partial_{1} U_{a}(x(a), y(a))=0$ by (11), this implies (15), and we conclude that (13) holds. As noted earlier, this, in turn, establishes that $a^{*}<z_{A}<b^{*}$. Repeating the same arguments for $b^{*}$ we also find $a^{*}<z_{B}<b^{*}$ in the analogous way, and hence the theorem.

Proof of Theorem 2. We begin by observing that $B_{z_{B}}$ is a strictly increasing function on $[0,1]$ (as shown within the proof of Lemma 1). Thus, it is invertible on the set $B_{z_{B}}([0,1])$; we denote the associated inverse function by $C_{z_{B}}$. Clearly, 
$C_{z_{B}}$ is differentiable everywhere on $B_{z_{B}}([0,1])$, and its derivative is continuous everywhere but at $B_{z_{B}}\left(z_{B}\right)$.

Consider any $\left(x^{*}, y^{*}\right) \in \mathcal{N}\left(a^{*}, b^{*}\right)$. We claim that

$$
x^{*}<B_{z_{A}}\left(y^{*}\right)
$$

and

$$
\left.B_{z_{B}}\left(x^{*}\right)<y^{*} \quad \text { (that is, } x^{*}<C_{z_{B}}\left(y^{*}\right)\right) .
$$

In what follows, we shall only supply the arguments needed to establish (16), the necessary arguments for (17) are similar and hence omitted.

Let $x:=B_{z_{A}}\left(y^{*}\right)$. If $x^{*} \leq z_{A}$ and $y \neq z_{A}$,we have $x^{*} \leq z_{A}<x$ by (3). If $x^{*} \leq z_{A}$ and $y^{*}=z_{A}$, then by Lemma 1 and (3), we get $x^{*}<y^{*}=z_{A}=x$. The nontrivial case to consider is, then, the case in which $x^{*}>z_{A}$. To deal with this case, recall that the first order conditions yield

$$
u_{a^{*}}\left(x^{*}\right)-u_{a^{*}}\left(y^{*}\right)+\left(x^{*}+y^{*}\right) u_{a^{*}}^{\prime}\left(x^{*}\right)=0
$$

and

$$
u_{z_{A}}(x)-u_{z_{A}}\left(y^{*}\right)+\left(x+y^{*}\right) u_{z_{A}}^{\prime}(x)=0 .
$$

To prove (16), then, it is enough to show that the left hand side of (19) must be strictly positive for $x \leq x^{*}$. Since $u_{z_{A}}$ is strictly decreasing and concave, it is easily seen that if this is the case for $x=x^{*}$, it must be so for $x<x^{*}$ as well. Hence, (16) will be established if we can show that

$$
u_{z_{A}}\left(x^{*}\right)-u_{z_{A}}\left(y^{*}\right)+\left(x^{*}+y^{*}\right) u_{z_{A}}^{\prime}\left(x^{*}\right)>0 .
$$

Solving for $x^{*}+y^{*}$ in (18) and substituting the resulting expression in (20), and then dividing both sides by $u^{\prime}\left(x^{*}-z_{A}\right)$, we find that (20) holds if and only if

$$
T:=\frac{u\left(x^{*}-z_{A}\right)-u\left(y^{*}-z_{A}\right)}{u^{\prime}\left(x^{*}-z_{A}\right)}-\frac{u\left(x^{*}-a^{*}\right)-u\left(y^{*}-a^{*}\right)}{u^{\prime}\left(x^{*}-a^{*}\right)}<0 .
$$

Now define the real map $\phi: \mathbf{R}_{+}^{2} \rightarrow \mathbf{R}$ by

$$
\phi(t, h):=\frac{u\left(x^{*}-z_{A}+h\right)-u(t+h)}{u^{\prime}\left(x^{*}-z_{A}+h\right)}
$$

and use Assumption [A1] to show that $\frac{\partial}{\partial h} \phi(t, h)>0$. (This step is analogous to the analysis of the function $H$ introduced in the proof of Theorem 1.) But then, since $z_{A}-a^{*}>0$ by Theorem 1 ,

$$
T=\phi\left(y^{*}-z_{A}, 0\right)-\phi\left(y^{*}-z_{A}, z_{A}-a^{*}\right)<0
$$

which yields (21) and hence (16).

Now let $\left\{\left(x^{\prime}, y^{\prime}\right)\right\}=\mathcal{N}\left(z_{A}, z_{B}\right)$. By the mean value theorem, and (16) and (17), we get

$$
x^{*}-x^{\prime}<B_{z_{A}}\left(y^{*}\right)-x^{\prime}=B_{z_{A}}\left(y^{*}\right)-B_{z_{A}}\left(y^{\prime}\right)=B_{z_{A}}^{\prime}(w)\left(y^{*}-y^{\prime}\right)
$$


and

$$
x^{*}-x^{\prime}<C_{z_{B}}\left(y^{*}\right)-x^{\prime}=C_{z_{B}}\left(y^{*}\right)-C_{z_{B}}\left(y^{\prime}\right)=C_{z_{B}}^{\prime}\left(w^{\prime}\right)\left(y^{*}-y^{\prime}\right)
$$

for some $w, w^{\prime} \in\left[\min \left\{y^{*}, y^{\prime}\right\}, \max \left\{y^{*}, y^{\prime}\right\}\right]$. If $y \leq y^{\prime}$, then by (23) and the fact that $C_{z_{B}}^{\prime}\left(w^{\prime}\right) \geq 1$, we obtain $x-x^{\prime}<y-y^{\prime}$. If $y>y^{\prime}$, then by (22) and the fact that $B_{z_{A}}^{\prime}(w) \leq 1$, we again get $x-x^{\prime}<y-y^{\prime}$. We thus conclude that $x-y<x^{\prime}-y^{\prime}$ and the proof is complete.

Proofs of Theorems 3 and 4. While the arguments become considerably more tedious, Theorems 3 and 4 are proved in precisely the same way we have proved Theorems 1 and 2 above. For brevity, therefore, we omit these proofs here. The detailed arguments can be obtained from any one of the authors upon request.

\section{References}

1. Alesina, A. Rosenthal, H.: Partisan politics, divided government, and the economy. New York: Cambridge University Press 1995

2. Alesina, A., Rosenthal, H.: A theory of divided government. Econometrica 64, 1311-1343 (1996)

3. Alesina, A., Rosenthal, H.: Polarized platforms and moderate policies with checks and balances. Journal of Public Economics 75, 1-20 (2000)

4. Barlow R. E., Proschan, F.: Statistical theory of reliability and life testing. Silver Spring, MD: To Begin With 1981

5. Baron, D. P.: Electoral competition with informed and uninformed voter. American Political Science Review 88, 33-47 (1994)

6. Blomberg, S. B., Harrington, J.E.: A theory of rigid extremists and flexible moderates with an application to the U.S. Congress. American Economic Review 90, 605-620 (2000)

7. Calvert, R. L.: Robustness of the multidimensional voting model: candidates' motivations, uncertainty and convergence. American Journal of Political Science 29, 69-95 (1985)

8. Caplin, A., Nalebuff, B.: Aggregation and social choice: a mean voter theorem. Econometrica 59, 1-23 (1991)

9. Feddersen, T.: A voting model implying Duverger's Law and positive turnout. American Journal of Political Science 36, 938-962 (1992)

10. Fershtman, C., Judd, K.L.: Equilibrium incentives in oligopoly. American Economic Review 77, 927-940 (1987)

11. Fershtman, C., Kalai, E.: Unobserved delegation. International Economic Review 38, 763-778 (1997)

12. Gerber, E., Morton, R.: Primary election systems and representation. Journal of Law, Economics, and Organization 14, 304-324 (1998)

13. Ghosh, P.: Electoral competition, moderating institutions and political extremism. Mimeo, UBC (1999)

14. Katz, M.: Game-playing agents: Unobservable contracts as precommitments. Rand Journal of Economics 22, 307-328 (1991)

15. Koçkesen, L., Ok, E. A.: Strategic delegation by unobservable incentive contracts. Mimeo, NYU (1999)

16. Koçkesen, L., Ok, E. A., Sethi, R.: The strategic advantage of negatively interdependent preferences. Journal of Economic Theory 92, 274-299 (2000)

17. Ortuño-Ortin, I.: A spatial model of political competition and proportional representation. Social Choice and Welfare 14, 427-438 (1997)

18. Poole, K. T., Rosenthal, H.: U.S. presidential elections 1968-1980: A spatial analysis. American Journal of Political Science 29, 357-384 (1984)

19. Poole, K. T., Rosenthal, H.: Patterns of congressional voting. American Journal of Political Science 35, 228-278 (1991) 
20. Roemer, J. E. : A theory of policy differentiation in single issue electoral politics. Social Choice and Welfare 11, 355-380 (1994)

21. Roemer, J. E.: The democratic political economy of progressive taxation. Econometrica 67, 1-20 (1999)

22. Sklivas, S.: The strategic choice of managerial incentives. Rand Journal of Economics 18, 452-458 (1987)

23. Weber, S.: On hierarchical spatial competition. Review of Economic Studies 59, 407-425 (1992)

24. Wittman, D. A.: Candidates with policy preferences: a dynamic model. Journal of Economic Theory 14, 180-189 (1997)

25. Wittman, D. A.: Candidate motivation: a synthesis of alternatives. American Political Science Review 77, 142-157 (1983) 